hep-ph/9611302

ILL-(TH)-96-13

November 1996

\title{
Supersymmetric QCD correction to top-quark production at the Tevatron
}

\author{
Zack Sullivan \\ Department of Physics \\ University of Illinois \\ 1110 West Green Street \\ Urbana, IL 61801
}

\begin{abstract}
We calculate the supersymmetric QCD correction to top-quark production at the Fermilab Tevatron, allowing for arbitrary left-right mixing of the squarks. We find that the correction is significant for several combinations of gluino and squark masses, e.g. $+33 \%$ for $m_{g}=200 \mathrm{GeV}, m_{\tilde{t}}=m_{q}=75 \mathrm{GeV}$.
\end{abstract}




\section{Introduction}

The discovery of the top quark [1] provides a unique opportunity to search for effects beyond the Standard Model. The top quark mass $m_{t}=175 \pm 6 \mathrm{GeV}$ has been measured to $3.5 \%$, and the cross section has been measured to $\approx 25 \%$ [2]. With the copious production of top quarks in Run II of the Fermilab Tevatron and future upgrades, the cross section will be measured to within $6 \%$ with $10 \mathrm{fb}^{-1}$ of data [3]. Comparison of the theoretical cross section to that measured will test the Standard Model and may indicate the presence of new physics.

Supersymmetry is a promising candidate for new physics. Currently, only lower bounds on the masses of the superpartners have been set. Barring discovery, direct searches for SUSY will eliminate a small range of parameter space, since these searches depend strongly on the modelling of the decays of the supersymmetric particles. In contrast, some effects of virtual supersymmetry are less model dependent, thus extending the reach of experiment. If virtual SUSY effects are found to be large enough, an indirect search may provide the first sign of supersymmetry. In this paper we calculate the supersymmetric QCD correction to $t \bar{t}$ production at the Fermilab Tevatron.

Direct searches for SUSY are generally performed separately for top squarks, the light quark superpartners, and gluinos. This is motivated by minimal supergravity models which argue that all scalar particles acquire a mass on the order of the SUSY breaking scale [4]. A heavy top quark loop dominates the running of the masses to low energies, forcing the mass of the two top squarks below that of the rest of the squarks. Additionally, mixing of the left-right weak eigenstates of the top squarks may result in the top squark $\widetilde{t}_{1}$ becoming the lightest squark []. The lightest top squark has a mass limit of $m_{\widetilde{t}_{1}}>47 \mathrm{GeV}$ [6]. The mass limit of the gluino is $m_{g}>154 \mathrm{GeV}$, when the light quark superpartners are assumed to be heavier than the gluino [7]. Current experimental limits are extracted assuming specific values of the SUSY parameters, and may be relaxed [8]. Other regions of parameter space have been eliminated [9, 10], but these limits are generally model dependent. Exhaustive direct searches will reach $300 \mathrm{GeV}$ for gluinos and $100 \mathrm{GeV}$ for top squarks with $10 \mathrm{fb}^{-1}$ of data at the Tevatron [3]. Until the advent of the CERN Large Hadron Collider, the presence of heavier SUSY particles will only be suggested by their effects on Standard Model processes.

The NLO QCD cross section for $t \bar{t}$ production with resummed gluon emission at a $\sqrt{S}=2 \mathrm{TeV} p \bar{p}$ collider has been calculated [11]. The dominant mechanism of top-quark production at the Tevatron is $q \bar{q}$ annihilation. It is expected that the dominant SUSY contribution to top production will be in the form of QCD corrections to this process. We consider the SUSY correction to the cross section as a correction to the dominant process as shown in Fig. 1. The calculation of the SUSY correction to top-quark production is different from typical SUSY calculations in that the number of assumptions about supersymmetry necessary to predict phenomenologically interesting results is minimal. It is assumed that R-parity is conserved so that the interaction terms in the Lagrangian are the simple supersymmetrization of the Standard Model interactions. No assumptions about the mechanism of SUSY breaking or of unification are required. In a strong-interaction process, the correction depends only on the observed masses of the gluino and squarks, and the mixing angle that relates the squark mass eigenstates to their interaction eigenstates. For the purpose of this calculation, and in order to cover the greatest range of models, we treat top squarks $\widetilde{t}$ separately from the light-quark superpartners $\widetilde{q}$. We present analytic and numerical results for degenerate squark masses, and for the case where the top squarks are light compared to the light-quark superpartners, the 'heavy squarks'. Results for $m_{\widetilde{t}}, m_{\widetilde{q}}>50 \mathrm{GeV}, m_{\tilde{g}}>150 \mathrm{GeV}$, and arbitrary left-right mixing of the squarks are presented.

The SUSY QCD correction to top production in $e^{+} e^{-}$annihilation has been studied in Ref. 12. The correction in $p \bar{p}$ annihilation has been presented in Refs. 13, 14, 15] for the case of degenerate squark masses. The calculations of Refs. [13, 14] neglect the contribution of the vacuum polarization and the crossed-box diagram, which arises because the gluino is a Majorana particle. In addition, Ref. [14 assumes that the box contribution may be ignored. We find that these contributions are numerically significant, as demonstarted in Sec. III A. Our results are numerically comparable to Ref. [15], however there is an important sign discrepancy between the two box terms that we discuss in Sec. II. There also appears to be a misprint in the analytic results of that paper that we describe at the end of our Appendix. We provide a complete calculation of the SUSY correction to the cross section for arbitrary masses and top-squark mixing, and discuss the phenomenological significance of the result. In addition, we discuss the tenability of finding SUSY thresholds in $t \bar{t}$ invariant mass distributions. Finally we address the issue of parity violation in a supersymmetric strong force. 
This paper is organized as follows. In Section II, we present the analytic form of the $\mathcal{O}\left(\alpha_{s}\right)$ SUSY QCD correction to the $p \bar{p} \rightarrow t \bar{t}$ cross section. In Section III A, we remark on the relative size of the terms in the correction. We present numerical results for the correction to the $p \bar{p} \rightarrow t \bar{t}$ cross section in Sec. III B. In Section III C, we show $t \bar{t}$ invariant mass distributions for several choices of gluino mass. We discuss the size of the strong force parity-violating left-right asymmetry in Sec. III D. Conclusions are presented in Sec. IV. We present analytic expressions for the vacuum, vertex and box terms in the Appendix.

\section{Analytic Supersymmetric QCD Correction}

The one-loop supersymmetric QCD contribution to the $q \bar{q} \rightarrow t \bar{t}$ cross section at leading order in $\alpha_{s}$ is attributed to the cross term in the matrix element between the tree level diagram and the one-loop diagrams presented in Fig. 1. The general form of the vertex corrections, consistent with current conservation, is

$$
\begin{aligned}
& i \bar{u}\left(p_{1}\right) \Gamma^{\mu, A} v\left(p_{2}\right)=-i g_{s}\left[\bar{u}\left(p_{1}\right) T^{A} \gamma^{\mu} v\left(p_{2}\right)\right. \\
& \left.\quad-\frac{\alpha_{s}}{4 \pi} \bar{u}\left(p_{1}\right) T^{A}\left[V \gamma^{\mu}+S\left(p_{1}^{\mu}-p_{2}^{\mu}\right) / m_{q}+A\left(\gamma^{\mu} q^{2}-2 m_{q} q^{\mu}\right) \gamma_{5}\right] v\left(p_{2}\right)\right]
\end{aligned}
$$

where $p_{1}$ and $p_{2}$ are the momenta of the quark and antiquark, $q=p_{1}+p_{2}, T^{A}$ is a $S U(3)$ generator, and $V$, $S$, and $A$ are the vector, scalar, and anapole form factors, respectively. The analytic forms of $V, S, A$, the gluon vacuum polarization $\Pi$, and the corrections due to the box and crossed-box diagrams, $B$ and $C$, are given in an appendix. The anapole term $A$ does not contribute to the total cross section at this order in the expansion. It is used in Sec. III D, however, in determining the parity-violating left-right asymmetry due to the squark mixing. The Dirac algebra and loop integrals were evaluated using dimensional regularization. The analytic cross section was derived in the $\overline{M S}$ renormalization scheme. The Feynman rules for the SUSY verticies were derived from Ref. [16] for the physically-relevant mass eigenstates of the squarks rather than the interaction eigenstates. Mixing of the squarks is therefore explicit and parameterized by mixing angles $\theta_{\widetilde{t}}$ and $\theta_{\widetilde{q}}$ for the stops and light-quark superpartners, respectively:

$$
\begin{aligned}
& \left(\begin{array}{l}
\widetilde{t}_{1} \\
\widetilde{t}_{2}
\end{array}\right)=\left(\begin{array}{rr}
\cos \theta_{\widetilde{t}} & \sin \theta_{\widetilde{t}} \\
-\sin \theta_{\tilde{t}} & \cos \theta_{\tilde{t}}
\end{array}\right)\left(\begin{array}{c}
\widetilde{t}_{L} \\
\widetilde{t}_{R}
\end{array}\right) \\
& \left(\begin{array}{c}
\widetilde{q}_{1} \\
\widetilde{q}_{2}
\end{array}\right)=\left(\begin{array}{rr}
\cos \theta_{\widetilde{q}} & \sin \theta_{\widetilde{q}} \\
-\sin \theta_{\widetilde{q}} & \cos \theta_{\widetilde{q}}
\end{array}\right)\left(\begin{array}{c}
\widetilde{q}_{L} \\
\widetilde{q}_{R}
\end{array}\right) .
\end{aligned}
$$

The spin- and color-averaged parton-level differential cross section is given by

$$
\frac{d \widehat{\sigma}}{d z}=\frac{\beta}{32 \pi \widehat{s}}|M|^{2},
$$

where $z$ is the cosine of the angle between the incoming quark and the top quark, $\beta=\sqrt{1-4 m_{t}^{2} / \widehat{s}}$, and $\sqrt{\widehat{s}}$ is the parton center-of-momentum energy. The Born matrix element squared is given by

$$
\left|M_{0}\right|^{2}=\frac{32 \pi^{2} \alpha_{s}^{2}}{9}\left[2-\beta^{2}\left(1-z^{2}\right)\right] .
$$

Integrating over $-1 \leq z \leq 1$ readily yields the Born-level cross section

$$
\widehat{\sigma}_{0}=\frac{4 \pi \alpha_{s}^{2} \beta}{9 \widehat{s}}\left(1-\beta^{2} / 3\right) .
$$

The correction arises from the cross term in the square of the amplitude. This correction is the sum of the terms:

$$
2 \operatorname{Re}\left[M_{0}^{\dagger} M_{\Pi}\right]=-\frac{\alpha_{s}}{2 \pi}\left|M_{0}\right|^{2} \operatorname{Re}[\Pi(\widehat{s})-\Pi(0)]
$$




$$
\begin{aligned}
2 \operatorname{Re}\left[M_{0}^{\dagger} M_{V}\right] & =-\frac{\alpha_{s}}{2 \pi}\left|M_{0}\right|^{2} \operatorname{Re}[V] \\
2 \operatorname{Re}\left[M_{0}^{\dagger} M_{S}\right] & =\frac{32 \pi \beta^{2} \alpha_{s}^{3}}{9}\left(1-z^{2}\right) \operatorname{Re}[S] \\
2 \operatorname{Re}\left[M_{0}^{\dagger} M_{B O X}\right] & =\frac{32 \pi \alpha_{s}^{3}}{9 \widehat{s}} \operatorname{Re}\left[\frac{7}{3} B+\frac{2}{3} C\right] .
\end{aligned}
$$

We renormalize the vacuum polarization correction so that it corresponds to the known value of $\alpha_{s}$ in the $\overline{M S}$ scheme at low energy. The integration over phase space is trivial except for the box and crossed-box matrix elements, $B$ and $C$, which depend implicitly on $z$. The relative sign between the box and crossed-box terms should be noted. The color factor associated with $C$ is $-2 / 3$. However, Fermi statistics, and the proper ordering of the Dirac indicies of the gluino fields in the amplitude, provide a non-trivial relative sign difference between the two diagrams. The net result is that the two contributions constructively interfere. This disagrees with the calculation of Ref. [15], which claims that the terms destructively interfere.

The total cross section for top production in $p \bar{p}$ annihilation is obtained by convolving the parton cross section for annihilation into a $t \bar{t}$ final state with the parton distribution functions of the proton and antiproton. The integral may be parameterized as

$$
\sigma=\int_{4 m_{t}^{2} / S}^{1} d \tau \widehat{\sigma}(\tau S) \int_{\ln (\tau) / 2}^{-\ln (\tau) / 2} d \eta P\left(\sqrt{\tau} e^{\eta}, \sqrt{\widehat{s}}\right) \bar{P}\left(\sqrt{\tau} e^{\eta}, \sqrt{\widehat{s}}\right)
$$

where $\sqrt{S}=2 \mathrm{TeV}, \tau=\widehat{s} / S$ and $P\left(x_{1}, \mu\right), \bar{P}\left(x_{2}, \mu\right)$ are the proton and antiproton parton distribution functions (PDF's).

In the following section, numerical results are presented for a top quark of mass $m_{t}=175 \mathrm{GeV}$. Analytic expressions were reduced to scalar n-point integrals [17] and evaluated with the aid of the code FF [18] in order to ensure numerical stability. For those cases that FF does not handle, the analytic solutions to the integrals were substituted. The integrals were evaluated using both the MRS(A') [19] and CTEQ3M [20] PDF's. The coupling $\alpha_{s}$ was evaluated as in the PDF's in order to be consistent. Nearly identical results were obtained using both sets, therefore, only the results obtained using the MRS(A') PDF's are presented.

\section{Numerical Results}

\section{A. Relative Size of the Correction Terms}

In Figure 2 we show the correction to the total cross section as a function of common squark mass $m_{\widetilde{Q}} \equiv m_{\tilde{q}}=m_{\widetilde{t}}$, for $m_{\tilde{g}}=200 \mathrm{GeV}$. The contribution of the vacuum, vector, scalar, and box terms are shown separately. The total correction is also shown for comparison. The box diagrams give the largest contribution to the cross section for $m_{\widetilde{Q}}<110 \mathrm{GeV}$, and are significant for $m_{\widetilde{Q}}<400 \mathrm{GeV}$. This invalidates the assumption of Ref. [14] that the box terms may be neglected over the range of masses they investigated. Similarly it contradicts the conclusion of Ref. 15] that the contribution of the box terms is small. The vacuum correction, that was ignored in Refs. [13, 14], also plays an important role. The gluino loops in the vacuum polarization give a constant negative correction when the squarks decouple. When $m_{\widetilde{Q}}=1 \mathrm{TeV}$, the correction is seen to come almost entirely from the vacuum polarization. The contribution of the scalar term $S$ is negligible. It first appears at this order in the final state correction, and is suppressed relative to the other terms by a power of the top-quark mass. The decoupling of the vector, scalar, and box terms is evident in Fig. 2, as the corrections decrease when the squark mass increases.

\section{B. $t \bar{t}$ Cross Section}

The correction to the $p \bar{p} \rightarrow t \bar{t}$ cross section is shown in Fig. 3 as a function of the gluino mass for a wide range of degenerate squark masses $m_{\widetilde{Q}}$, where $m_{\widetilde{Q}} \equiv m_{\tilde{q}}=m_{\widetilde{t}}$. As expected from decoupling, the magnitude of the correction decreases as the squark mass increases. Squarks of mass $50 \mathrm{GeV}$ set the range of the correction from $-11.8 \%$ for a gluino of $150 \mathrm{GeV}$ to $+44 \%$ for a gluino of $200 \mathrm{GeV}$. The correction 
changes sign as $m_{\tilde{g}}$ approaches $m_{t}$. The correction changes rapidly as the threshold for gluino production moves through the top-quark threshold. Note that the correction is nearly independent of gluino mass when $m_{g}>700 \mathrm{GeV}$. In this region, the correction is entirely dominated by the squark vacuum terms and, to a lesser extent, the box terms.

In Figure 4 we show the correction to the total cross section as a function of degenerate squark mass $m_{\widetilde{Q}} \equiv m_{\tilde{q}}=m_{\widetilde{t}}$, for several gluino masses. Once $m_{\widetilde{Q}}>400 \mathrm{GeV}$, the correction becomes small and the squarks effectively decouple. In this region, the correction is dominated by the gluino vacuum terms. In Figs. 3 and 4 there is a large jump in the cross section when $m_{t}^{2}=m_{\widetilde{t}}^{2}+m_{\widetilde{g}}^{2}$. In Figure 4 , the correction jumps from $+6.5 \%$ to $-9.3 \%$ for $m_{\tilde{g}}=150 \mathrm{GeV}, m_{\tilde{t}}=90.1 \mathrm{GeV}$. This corresponds to a discontinuity in the real part of the $C_{0}$ scalar loop-integral in the final-state vertex correction 21. Such a discontinuity arises when the anomalous threshold crosses the real threshold for superpartner production in the complex $s$-plane [22].

The largest correction occurs when $m_{\tilde{g}}=200 \mathrm{GeV}$. This mass is used in Fig. 5 to show the correction as a function of heavy-squark mass $m_{\tilde{q}}$, for a variety of top-squark masses. This figure demonstrates that the correction is mostly influenced by the mass of the top squark. For example, the correction is $21 \%$ for $m_{\widetilde{t}}=50 \mathrm{GeV}$, and $m_{\tilde{q}}=300 \mathrm{GeV}$; whereas the correction is $16 \%$ for $m_{\widetilde{t}}=300 \mathrm{GeV}$, and $m_{\widetilde{q}}=50 \mathrm{GeV}$. Even if the heavy squarks decouple, the correction remains significant as long as $m_{\tilde{t}}<150 \mathrm{GeV}$.

In general, the left and right eigenstates of the squarks receive different corrections to their masses. This causes the mass of $\widetilde{Q}_{R}$ to be less than the mass of $\widetilde{Q}_{L}$. Top-squark masses are more effected by renormalization group running than the heavy-squark masses, because of the direct coupling of the top to the stops. Many analyses assume that the only light squark is $\widetilde{t}_{1}$, and look for top quarks decaying into them [10]. In Figure 6 we show the ratio of the correction at $\Delta m_{\widetilde{t}}=\left(m_{\widetilde{t}_{2}}-m_{\widetilde{t}_{1}}\right)$ to the correction at a common top-squark mass $\Delta m_{\tilde{t}}=0$, for $m_{\tilde{g}}=200 \mathrm{GeV}$ and $m_{\tilde{q}}=300 \mathrm{GeV}$. The ratio does not change by more than $2 \%$ for different values of $m_{\tilde{q}}$. We present three mixing angles, $\theta_{\widetilde{t}}=45^{\circ}, 90^{\circ}$, and $135^{\circ}$ that define the extremes of the mixing dependence of the correction. The form of the correction is $a+b \sin \left(2 \theta_{\tilde{t}}\right)$, thus the contribution of any mixing angle may be interpolated from the curves shown, where $\theta_{\widetilde{t}}=90^{\circ}$ is the central value. Note that if $\theta_{\tilde{t}}=135^{\circ}$, then the correction is nearly independent of $m_{\tau_{2}}$; whereas if $\theta_{\tilde{t}}=90^{\circ}$, where the mass eigenstates are the interaction eigenstates, the correction is roughly split between the two top squarks. To evaluate the correction for non-degenerate top-squark masses and top-squark mixing, multiply the ratio from Fig. 6 by the correction from Figs. 4 or 5. For example, the correction to top production is $7.7 \pm 0.1 \%$, when $m_{\widetilde{t}_{1}}=100 \mathrm{GeV}, m_{\widetilde{t}_{2}}=400 \mathrm{GeV}, m_{\tilde{g}}=200 \mathrm{GeV}, m_{\widetilde{q}}=400 \mathrm{GeV}$, and $\theta_{\widetilde{t}}=90^{\circ}$.

\section{C. $t \bar{t}$ Invariant Mass Distributions}

Since total cross section measurements are difficult to normalize, it is advantageous to look for deviations from the line-shapes predicted by the Standard Model. A sampling of the invariant mass of $t \bar{t}$ events provides another avenue to search for supersymmetry. In Figure 7 we show the total differential cross section as a function of $t \bar{t}$ invariant mass $M_{t \bar{t}}$, for gluinos of mass $m_{\tilde{g}}=150,175,200$, and $225 \mathrm{GeV}$. Several choices of degenerate squark mass $m_{\widetilde{Q}} \equiv m_{\widetilde{q}}=m_{\widetilde{t}}$, are presented. By looking for an excess in the invariant mass distribution, a gluino of mass between $175 \mathrm{GeV}$ and $225 \mathrm{GeV}$ may be observable.

There are two types of enhancement to the cross section that appear in Fig. 7. If $m_{\tilde{g}} \approx m_{t}$, the maximum of the invariant mass distribution is shifted toward the common threshold. This would also produce a steeper top-quark threshold region in the data. A singularity at the threshold for gluino pair production causes a cusp at $2 m_{\tilde{g}}$. The largest cusp occurs when $m_{\tilde{g}}=200 \mathrm{GeV}$, and $m_{\widetilde{Q}}=50 \mathrm{GeV}$. The amplitude of the cusp is $112 \%$ of the Standard Model differential cross section at this point. Despite the large normalization, the cusp will sit on a large continuum background. If we assume purely statistical errors, this cusp would appear at the $3 \sigma$ level with $3 \mathrm{fb}^{-1}$ of integrated luminosity. For $m_{\tilde{g}} \approx 200 \mathrm{GeV}$, the correction is most apparent for $m_{\widetilde{Q}}<150 \mathrm{GeV}$. If $m_{\tilde{g}}>225 \mathrm{GeV}$, then even with light squarks, the correction will be difficult to observe.

\section{Strong Force Parity Violation}

In the Standard Model, the top quark decays before its spin flips [23]. The helicity of the top quark is reflected in the angular distribution of the decay products of the $W$ boson in $t \rightarrow b W \rightarrow b \ell^{+} \nu$ and 
$t \rightarrow b W \rightarrow b \bar{d} u$ decays. (See Ref. 24] for a detailed account of the analyzing power of these decays.) The $g t \widetilde{t}$ interaction term in the SUSY Lagrangian treats left and right-handed top squarks differently. This leads to the interesting possibility of searching for parity violation in strong force interactions by analyzing the decay products in top-quark production.

An asymmetry in the number of left and right-handed top quarks arises in the production cross section when the top squarks have different masses. This asymmetry is given by

$$
\Delta \widehat{\sigma}_{A}=\widehat{\sigma}_{L}-\widehat{\sigma}_{R}=\frac{2 \beta^{2} \alpha_{s}^{3}}{27 \widehat{s}} \operatorname{Re}[A]
$$

where $\sigma_{L}, \sigma_{R}$ are the cross sections for the left and right helicities of the top quark. The measured left-right asymmetry $A_{L R}$ is the ratio of the integrated $\Delta \widehat{\sigma}_{A}$ to the total measured cross section

$$
A_{L R}=\frac{\Delta \sigma_{A}}{\sigma_{T O T}}=\frac{n_{L}-n_{R}}{n_{L}+n_{R}}
$$

where $n_{L}, n_{R}$ are the number of left and right-handed top quarks respectively. Unfortunately, we find that $A_{L R}$ is always less than $1 \%$ for any choice of the SUSY parameters. Therefore, if supersymmetric parity violation in the strong force exists, it will be very difficult to measure.

\section{Conclusions}

The supersymmetric QCD correction to the top-quark cross section, as measured at the Tevatron, has been calculated. We present analytic results for a minimal supersymmetric model that depends only on the masses of the superpartners and their mixing. We obtain numerical results for the total correction for all masses $m_{\tilde{g}}>150 \mathrm{GeV}, m_{\tilde{q}}>50 \mathrm{GeV}$ and $m_{\tilde{t}}>50 \mathrm{GeV}$. The correction is found to be large for gluino masses near $200 \mathrm{GeV}$. The correction is greater than $+10 \%$ for $m_{\tilde{g}}=200 \mathrm{GeV}$ and $m_{\tilde{q}}=m_{\tilde{t}}<190 \mathrm{GeV}$. If light top squarks $m_{\tilde{t}}<150 \mathrm{GeV}$ exist, then the correction should be observable with $10 \mathrm{fb}^{-1}$ at the Tevatron for $m_{\tilde{g}}<400 \mathrm{GeV}$, even if the heavy squarks decouple. If all of the squarks remain light, then the correction is significant even if the gluinos decouple. When considering a mass splitting between the top squarks, the mixing angle $\theta_{\tilde{t}}$ plays an important role. If $\theta_{\tilde{t}}$ is near $45^{\circ}$, or $135^{\circ}$, then the correction is almost entirely dependent on the mass of only one of the top squarks.

Should the gluino mass turn out to be near the current experimental limits, a gluino-pair threshold may be found near the top-quark production threshold. The advantage of looking for a cusp in the $t \bar{t}$ invariant mass distribution, is that the normalization of the top-quark cross section is not necessarily a limiting factor. Detector resolution effects and smearings will make this search very challenging. It is reasonable to expect that at least $10 \mathrm{fb}^{-1}$ of integrated luminosity would be required to find a cusp for the best case of $m_{\tilde{g}} \approx 200 \mathrm{GeV}$, and $m_{\widetilde{Q}}<150 \mathrm{GeV}$. Virtual SUSY thresholds are common in quark production [25]. A full detector-based analysis of these threshold regions would help determine the experimental significance of our results.

Parity violation in a purely strong force interaction arises in a supersymmetric Standard Model because the left- and right-handed top squarks interact differently. As long as the top-squark masses are different, an asymmetry in the number of left and right-handed top quarks will arise. Unfortunately, the effect is less than $1 \%$, and will be very difficult to measure.

\section{Acknowledgements}

The author is grateful to S. Willenbrock for his helpful comments and for suggesting this study, and for conversations with T. Stelzer. I gratefully acknowledge the support of a GAANN fellowship, under grant number DE-P200A40532, from the U. S. Department of Education.

\section{Appendix}

The form factors for the one-loop matrix elements in Eqn. 6 of the supersymmetric QCD correction to top-quark production are given below. The integrals are written in terms of n-point integrals [17], in the 
notation of FF [18]. For each appearance of a heavy squark $\widetilde{q}$, or top squark $\widetilde{t}$, the term should be summed with $\widetilde{q}_{1}$, or $\widetilde{t}_{1}$ first, and then $\pm \widetilde{q}_{2}$, or $\pm \widetilde{t}_{2}$ as indicated. The vacuum polarization is separated into terms proportional to the top squarks $\widetilde{t}$ and the heavy squarks $\widetilde{q}$.

$$
\begin{aligned}
\Pi= & \frac{1}{s}\left[\left(4 m_{\vec{g}}^{2}+2 s\right) B_{0}\left(m_{\vec{g}}^{2}, m_{\vec{g}}^{2}, s\right)-4 A_{0}\left(m_{\vec{g}}^{2}\right)+\frac{2}{3}\left[A_{0}\left(m_{\widetilde{t}}^{2}\right)+5 A_{0}\left(m_{\widetilde{q}}^{2}\right)\right]\right. \\
+ & \frac{1}{6}\left(s-4 m_{\widetilde{t}}^{2}\right) B_{0}\left(m_{\widetilde{t}}^{2}, m_{\widetilde{t}}^{2}, s\right)+\frac{5}{6}\left(s-4 m_{\widetilde{q}}^{2}\right) B_{0}\left(m_{\widetilde{q}}^{2}, m_{\widetilde{q}}^{2}, s\right) \\
& \left.-4 s \Delta+4 m_{\vec{g}}^{2}+\frac{2}{3}\left(s-m_{\widetilde{t}}^{2}-5 m_{\widetilde{q}}^{2}\right)\right]
\end{aligned}
$$

The initial- and final-state vertex correction form factors have the same functional form. For the initial state, $m_{q}=0$, and $m_{\widetilde{Q}}=m_{\widetilde{q}}$. For the final state, $m_{q}=m_{t}$, and $m_{\widetilde{Q}}=m_{\widetilde{t}}$. The two squarks $\widetilde{Q}_{1}$ and $\pm \widetilde{Q}_{2}$ are summed as before. Arbitrary mixing is allowed for both the top squarks and the heavy squarks. The 3 -point integrals have the form

$$
\begin{aligned}
& C\left(m_{1}^{2}, m_{2}^{2}, m_{3}^{2}\right)=C\left(m_{1}^{2}, m_{2}^{2}, m_{3}^{2}, m_{q}^{2}, m_{q}^{2}, s\right) \\
& V=- \frac{3}{2}\left[m_{q}^{2} C_{21}\left(m_{\widetilde{g}}^{2}, m_{\widetilde{Q}}^{2}, m_{\widetilde{g}}^{2}\right)+m_{q}^{2} C_{22}\left(m_{\widetilde{g}}^{2}, m_{\widetilde{Q}}^{2}, m_{\widetilde{g}}^{2}\right)+\left(s-2 m_{q}^{2}\right) C_{23}\left(m_{\widetilde{g}}^{2}, m_{\widetilde{Q}}^{2}, m_{\widetilde{g}}^{2}\right)\right. \\
&+2 C_{24}\left(m_{\widetilde{g}}^{2}, m_{\widetilde{Q}}^{2}, m_{\widetilde{g}}^{2}\right)-1+2 m_{q}^{2} C_{11}\left(m_{\widetilde{g}}^{2}, m_{\widetilde{Q}}^{2}, m_{\widetilde{g}}^{2}\right)+\left(s-2 m_{q}^{2}\right) C_{12}\left(m_{\widetilde{g}}^{2}, m_{\widetilde{Q}}^{2}, m_{\widetilde{g}}^{2}\right) \\
&\left.-m_{\widetilde{g}}^{2} C_{0}\left(m_{\widetilde{g}}^{2}, m_{\widetilde{Q}}^{2}, m_{\widetilde{g}}^{2}\right) \pm 2 m_{q} m_{\widetilde{g}} \sin \left(2 \theta_{\widetilde{Q}}\right) C_{0}\left(m_{\widetilde{g}}^{2}, m_{\widetilde{Q}}^{2}, m_{\widetilde{g}}^{2}\right)\right]+\frac{1}{3} C_{24}\left(m_{\widetilde{Q}}^{2}, m_{\widetilde{g}}^{2}, m_{\widetilde{Q}}^{2}\right) \\
&+\frac{4}{3}\left[-B_{1}\left(m_{\widetilde{Q}}^{2}, m_{\widetilde{g}}^{2}, m_{q}^{2}\right)+\left(m_{q}^{2}+m_{\widetilde{g}}^{2}-m_{\widetilde{Q}}^{2}\right) B_{0}^{\prime}\left(m_{\widetilde{Q}}^{2}, m_{\widetilde{g}}^{2}, m_{q}^{2}\right) \mp 2 m_{q} m_{\widetilde{g}} \sin \left(2 \theta_{\widetilde{Q}}\right) B_{0}^{\prime}\left(m_{\widetilde{Q}}^{2}, m_{\widetilde{g}}^{2}, m_{q}^{2}\right)\right] \\
& S= {\left[m_{q}^{2} C_{22}\left(m_{\widetilde{g}}^{2}, m_{\widetilde{Q}}^{2}, m_{\widetilde{g}}^{2}\right)-m_{q}^{2} C_{23}\left(m_{\widetilde{g}}^{2}, m_{\widetilde{Q}}^{2}, m_{\widetilde{g}}^{2}\right) \mp m_{q} m_{\widetilde{g}} \sin \left(2 \theta_{\widetilde{Q}}\right) C_{12}\left(m_{\widetilde{g}}^{2}, m_{\widetilde{Q}}^{2}, m_{\widetilde{g}}^{2}\right)\right] } \\
&-\frac{1}{3}\left[-m_{q}^{2} C_{22}\left(m_{\widetilde{Q}}^{2}, m_{\widetilde{g}}^{2}, m_{\widetilde{Q}}^{2}\right)+m_{q}^{2} C_{23}\left(m_{\widetilde{Q}}^{2}, m_{\widetilde{g}}^{2}, m_{\widetilde{Q}}^{2}\right) \mp m_{q} m_{\widetilde{g}} \sin \left(2 \theta_{\widetilde{Q}}\right)\left[C_{12}\left(m_{\widetilde{Q}}^{2}, m_{\widetilde{g}}^{2}, m_{\widetilde{Q}}^{2}\right)\right.\right. \\
&\left.\left.+\frac{1}{2} C_{0}\left(m_{\widetilde{Q}}^{2}, m_{\widetilde{g}}^{2}, m_{\widetilde{Q}}^{2}\right)\right]\right] \\
& A= \frac{3}{2 s} \cos \left(2 \theta_{\widetilde{Q}}\right)\left[m_{q}^{2}\left[C_{21}\left(m_{\widetilde{g}}^{2}, m_{\widetilde{Q}}^{2}, m_{\widetilde{g}}^{2}\right)+C_{22}\left(m_{\widetilde{g}}^{2}, m_{\widetilde{Q}}^{2}, m_{\widetilde{g}}^{2}\right)\right]+\left(s-2 m_{q}^{2}\right) C_{23}\left(m_{\widetilde{g}}^{2}, m_{\widetilde{Q}}^{2}, m_{\widetilde{g}}^{2}\right)\right. \\
&+2 C_{24}\left(m_{\widetilde{g}}^{2}, m_{\widetilde{Q}}^{2}, m_{\widetilde{g}}^{2}\right)+s C_{12}\left(m_{\widetilde{g}}^{2}, m_{\widetilde{Q}}^{2}, m_{\widetilde{g}}^{2}\right)-m_{\widetilde{g}}^{2} C_{0}\left(m_{\widetilde{g}}^{2}, m_{\widetilde{Q}}^{2}, m_{\widetilde{g}}^{2}\right)-\frac{2}{9} C_{24}\left(m_{\widetilde{Q}}^{2}, m_{\widetilde{g}}^{2}, m_{\widetilde{Q}}^{2}\right) \\
&\left.+\frac{8}{9} B_{1}\left(m_{\widetilde{Q}}^{2}, m_{\widetilde{g}}^{2}, m_{q}^{2}\right)\right]
\end{aligned}
$$

The 4-point integrals in the box terms are

$$
\begin{aligned}
D & =D\left(m_{\vec{g}}^{2}, m_{\widetilde{q}}^{2}, m_{\widetilde{g}}^{2}, m_{\vec{t}}^{2}, 0,0, m_{t}^{2}, m_{t}^{2}, s, m_{t}^{2}-2 p_{1} \cdot p_{3}\right) \\
D^{c} & =D^{c}\left(m_{\vec{g}}^{2}, m_{\vec{q}}^{2}, m_{\vec{g}}^{2}, m_{\widetilde{t}}^{2}, 0,0, m_{t}^{2}, m_{t}^{2}, s, m_{t}^{2}-2 p_{2} \cdot p_{3}\right),
\end{aligned}
$$

where $p_{1} \cdot p_{3}=s(1-\beta z) / 4$ and $p_{2} \cdot p_{3}=s(1+\beta z) / 4$. The box and crossed-box terms are summed over each combination of squarks $\widetilde{q}_{i} \widetilde{t}_{j}$, where $i, j=1,2$. The mixing of the squarks is parameterized as

$$
a c+b d=\mp \frac{1}{4} \sin \left(2 \theta_{\mathfrak{t}}\right)
$$


for $\widetilde{t}_{1}$ and $\widetilde{t}_{2}$ respectively; and for $\widetilde{q}_{i} \widetilde{t}_{j}$

$$
\begin{gathered}
a^{2}+b^{2}=\frac{1}{4}\left[\cos ^{2}\left(\theta_{\widetilde{t}}-\theta_{\widetilde{q}}\right)+\cos ^{2}\left(\theta_{\tilde{t}}+\theta_{\widetilde{q}}\right)\right], i=j \\
c^{2}+d^{2}=\frac{1}{4}\left[\sin ^{2}\left(\theta_{\tilde{t}}-\theta_{\widetilde{q}}\right)+\sin ^{2}\left(\theta_{\widetilde{t}}+\theta_{\widetilde{q}}\right)\right], i=j \\
a^{2}+b^{2}=\frac{1}{4}\left[\sin ^{2}\left(\theta_{\widetilde{t}}-\theta_{\widetilde{q}}\right)+\sin ^{2}\left(\theta_{\widetilde{t}}+\theta_{\widetilde{q}}\right)\right], i \neq j \\
c^{2}+d^{2}=\frac{1}{4}\left[\cos ^{2}\left(\theta_{\widetilde{t}}-\theta_{\widetilde{q}}\right)+\cos ^{2}\left(\theta_{\widetilde{t}}+\theta_{\widetilde{q}}\right)\right], i \neq j . \\
B=m_{t} m_{\tilde{g}} s^{2}(a c+b d)\left[D_{11}-D_{12}+D_{13}+D_{0}\right]+m_{t}^{2} s^{2}\left(a^{2}+b^{2}\right)\left[-D_{12}-D_{23}-D_{24}\right. \\
\left.+D_{26}-2 D_{27} / s\right]+4 s\left(p_{2} \cdot p_{3}\right)^{2}\left(a^{2}+b^{2}\right)\left[-D_{12}+D_{13}-D_{24}+D_{25}-2 D_{27} / s\right] \\
+\left[m_{t}^{2} s+4\left(p_{1} \cdot p_{3}\right)^{2}\right]\left[m_{t}^{2}\left(a^{2}+b^{2}\right) D_{23}+m_{\widetilde{g}}^{2}\left(c^{2}+d^{2}\right) D_{0}-2 m_{t} m_{\widetilde{g}}(a c+b d) D_{13}\right] \\
C=m_{t} m_{\widetilde{g}} s^{2}(a c+b d)\left[D_{11}^{c}-D_{12}^{c}+D_{13}^{c}+D_{0}^{c}\right]+m_{t}^{2} s^{2}\left(c^{2}+d^{2}\right)\left[-D_{12}^{c}-D_{23}^{c}-D_{24}^{c}\right. \\
\left.+D_{26}^{c}-2 D_{27}^{c} / s\right]+4 s\left(p_{1} \cdot p_{3}\right)^{2}\left(c^{2}+d^{2}\right)\left[-D_{12}^{c}+D_{13}^{c}-D_{24}^{c}+D_{25}^{c}-2 D_{27}^{c} / s\right] \\
+\left[m_{t}^{2} s+4\left(p_{2} \cdot p_{3}\right)^{2}\right]\left[m_{t}^{2}\left(c^{2}+d^{2}\right) D_{23}^{c}+m_{\widetilde{g}}^{2}\left(a^{2}+b^{2}\right) D_{0}^{c}-2 m_{t} m_{\tilde{g}}(a c+b d) D_{13}^{c}\right]
\end{gathered}
$$

A few equations in the Appendix of Ref. 15] appear to be misprinted. As written they lead to divergent behavior that does not match Fig. 8 in that paper. With the following replacements, our analytic results agree up to the sign discrepency discussed in Sec. II.

$$
\begin{aligned}
F_{12}^{\mathrm{DB}} & =\frac{\alpha_{s}}{\pi}\left\{-\hat{s}\left(2 A_{5}^{\dagger} A_{5}^{\dagger}\right)\left[2 \hat{s} m_{t}^{2}+2\left(\hat{u}-m_{t}^{2}\right)^{2}\right]-m_{g} m_{t}\left(2 A_{5}^{\dagger} A_{5 x}\right)\left[2 \hat{s}^{2}\right]\right\} D_{12} \\
F_{13}^{\mathrm{DB}} & =\frac{\alpha_{s}}{\pi}\left\{\hat{s}\left(2 A_{5}^{\dagger} A_{5}^{\dagger}\right)\left[2\left(\hat{u}-m_{t}^{2}\right)^{2}\right]+m_{g}^{\sim} m_{t}\left(2 A_{5}^{\dagger} A_{5 x}\right)\left[2 \hat{s}\left(\hat{s}-2 m_{t}^{2}\right)-4\left(\hat{t}-m_{t}^{2}\right)^{2}\right]\right\} D_{13} \\
F_{12}^{\mathrm{CB}} & =\frac{\alpha_{s}}{\pi}\left\{-\hat{s}\left(2 A_{5}^{\dagger} A_{5}^{\dagger}\right)\left[2 \hat{s} m_{t}^{2}+2\left(\hat{t}-m_{t}^{2}\right)^{2}\right]-m_{g}^{\sim} m_{t}\left(2 \overline{A_{5}^{\dagger} A_{5 x}}\right)\left[2 \hat{s}^{2}\right]\right\} D_{12} \\
F_{13}^{\mathrm{CB}} & =\frac{\alpha_{s}}{\pi}\left\{\hat{s}\left(2 A_{5}^{\dagger} A_{5}^{\dagger}\right)\left[2\left(\hat{t}-m_{t}^{2}\right)^{2}\right]+m_{g} m_{t}\left(2 \overline{A_{5}^{\dagger} A_{5 x}}\right)\left[2 \hat{s}\left(\hat{s}-2 m_{t}^{2}\right)-4\left(\hat{u}-m_{t}^{2}\right)^{2}\right]\right\} D_{13}
\end{aligned}
$$




\section{References}

[1] F. Abe et al., CDF Collaboration, Phys. Rev. Lett. 74, 2626 (1995); S. Abachi et al., D0 Collaboration, Phys. Rev. Lett. 74, 2632 (1995).

[2] D. Gerdes, CDF Collaboration, FERMILAB-CONF-96/342-E. Proceedings 1996 DPF/DPB Summer Study, "New Directions for High Energy Physics," Snowmass, CO, June 25-July 12, 1996.

[3] "Future of Electroweak Physics at the Fermilab Tevatron: Report of the tev_2000 Study Group", edited by D. Amidei and R. Brock, FERMILAB-PUB-96/082, 1996.

[4] For reviews, see H. P. Nilles, Phys. Rep. 110, 1 (1984); P. Nath, R. Arnowitt, and A. Chamseddine, Applied N=1 Supergravity, ICTP series in Theoretical Physics, (World Scientific, Singapore, 1984); Also see references in J. Amundson et al., hep-ph/9609374. Proceedings of the 1996 DPF/DPB Summer Study, "New Directions for High Energy Physics," Snowmass, CO, June 25-July 12, 1996.

[5] J. Ellis and S. Rudaz, Phys. Lett. B128, 248 (1983); A. Bouquet, J. Kaplan and C. Savoy, Nucl. Phys. B262, 299 (1985).

[6] M. Schmitt (private communication), ALEPH preliminary results presented at the "LEP Jamboree", October 8, 1996.

[7] F. Abe et al., CDF Collaboration, Phys. Rev. Lett. 76, 2006 (1996).

[8] A. Datta, M. Guchait, and N. Parua, hep-ph/9609413 and references therein.

[9] S. Abachi et al., D0 Collaboration, FERMILAB-Conf-95/193-E, D0 Note 2614, 1995; H1 Collaboration, hep-ex/9605002.

[10] S. Abachi et al., D0 Collaboration, Phys. Rev. Lett. 76, 2222 (1996).

[11] P. Nason, S. Dawson, and R. K. Ellis, Nucl. Phys. B303, 607 (1988); Nucl. Phys. B327, 49 (1989); W. Beenakker, H. Kuijif, W. van Neerven, and J. Smith, Phys. Rev. D 40, 54 (1989); W. Beenakker, W. van Neerven, R. Meng, G. Schuler, and J. Smith, Nucl. Phys. B351, 507 (1991); E. Laenen, J. Smith, and W. van Neerven, Nucl. Phys. B369, 543 (1992); Phys. Lett. B321, 254 (1994); S. Catani, M. Mangano, P. Nason, and L. Trentadue, Phys. Lett. B378, 329 (1996); hep-ph/9604351; E. Berger and H. Contopanagos, Phys. Lett. B361, 115 (1995); Phys. Rev. D 54, 3085 (1996).

[12] A. Djouadi, M. Drees, and H. König, Phys. Rev. D 48, 3081 (1993).

[13] C. Li, B. Hu, J. Yang, and C. Hu, Phys. Rev. D 52, 5014 (1995); Erratum: Phys. Rev. D 53, 4112 (1996).

[14] J. Kim, J. Lopez, D. V. Nanopoulos, and R. Rangarajan, Phys. Rev. D 54, 4364 (1996).

[15] S. Alam, K. Hagiwara, and S. Matsumoto, Phys. Rev. D 55, 1307 (1997).

[16] H. Haber and G. Kane, Phys. Rep. 117, 75 (1985).

[17] G. Passarino and M. Veltman, Nucl. Phys. B160, 151 (1979).

[18] G. J. van Oldenborgh, Comput. Phys. Commun. 66, 1 (1991).

[19] A. D. Martin, W. J. Stirling, and R. G. Roberts, Phys. Rev. D 51, 4756 (1995).

[20] H. Lai, J. Botts, J. Huston, J. Morfin, J. Owens, J. Qiu, W.-K. Tung, and H. Weerts, Phys. Rev. D 51, 4763 (1995).

[21] G. 't Hooft and M. Veltman, Nucl. Phys. B153, 365 (1979).

[22] C. Itzykson and J. Zuber, Quantum Field Theory (McGraw-Hill, New York, 1980), p. 311. 
[23] I. Bigi, Y. Dokshitzer, V. Khoze, J. Kühn, and P. Zerwas, Phys. Lett. B181, 157 (1986).

[24] M. Jeżabek, Nucl. Phys. B (Proc. Suppl.) 37B, 197 (1994).

[25] J. Ellis and D. Ross, Phys. Lett. B383, 187 (1996). 


\section{Figure Captions}

Fig. 1: Feynman diagrams for the one-loop SUSY QCD correction to top quark production at the Tevatron. The first row contains the tree-level diagram. The second row contains the vacuum polarization correction to the gluon propagator due to squarks and gluinos. The third row contains the final state vertex correction and wave-function renormalization diagrams. The fourth row contains the initial state vertex correction and wave-function renormalization diagrams. The last row contains the box and crossed-box diagrams.

Fig. 2: Contribution of each term to the correction for $p \bar{p} \rightarrow t \bar{t}$ as a function of $m_{\tilde{q}}=m_{\widetilde{t}}$, for $m_{\tilde{g}}=$ $200 \mathrm{GeV}$.

Fig. 3: Change in the cross section for $p \bar{p} \rightarrow t \bar{t}$, as a function of gluino mass $m_{\widetilde{q}}$, for $m_{t}=175 \mathrm{GeV}$. Curves of constant degenerate squark mass $m_{\widetilde{q}}=m_{\widetilde{t}}$ are shown.

Fig. 4: Change in the cross section for $p \bar{p} \rightarrow t \bar{t}$, as a function of degenerate squark mass $m_{\tilde{q}}=m_{\widetilde{t}}$, for $m_{t}=175 \mathrm{GeV}$. Curves of constant gluino mass $m_{\tilde{g}}$ are shown.

Fig. 5: Change in the cross section for $p \bar{p} \rightarrow t \bar{t}$, as a function of heavy-squark mass $m_{\tilde{q}}$, for $m_{t}=175 \mathrm{GeV}$, and $m_{\tilde{q}}=200 \mathrm{GeV}$. Curves of constant top-squark mass $m_{\tilde{t}}$ are shown.

Fig. 6: The relative change to the correction is shown as a function of top-squark mass difference $\Delta m_{\widetilde{t}}=\left(m_{\widetilde{t}_{2}}-m_{\widetilde{t}_{1}}\right)$, for various $m_{\widetilde{t_{1}}}$, and mixing angles $\theta_{\widetilde{t}}$, with $m_{t}=175 \mathrm{GeV}$, and $m_{\tilde{g}}=200 \mathrm{GeV}$.

Fig. 7: Differential cross section for $p \bar{p} \rightarrow t \bar{t}$, as a function of $t \bar{t}$ invariant mass $M_{t \bar{t}}$, for $m_{t}=175 \mathrm{GeV}$. Figures are shown for $m_{\tilde{g}}=150,175,200$, and $225 \mathrm{GeV}$. Curves of constant degenerate squark mass $m_{\tilde{q}}=m_{\widetilde{t}}$ are shown. 


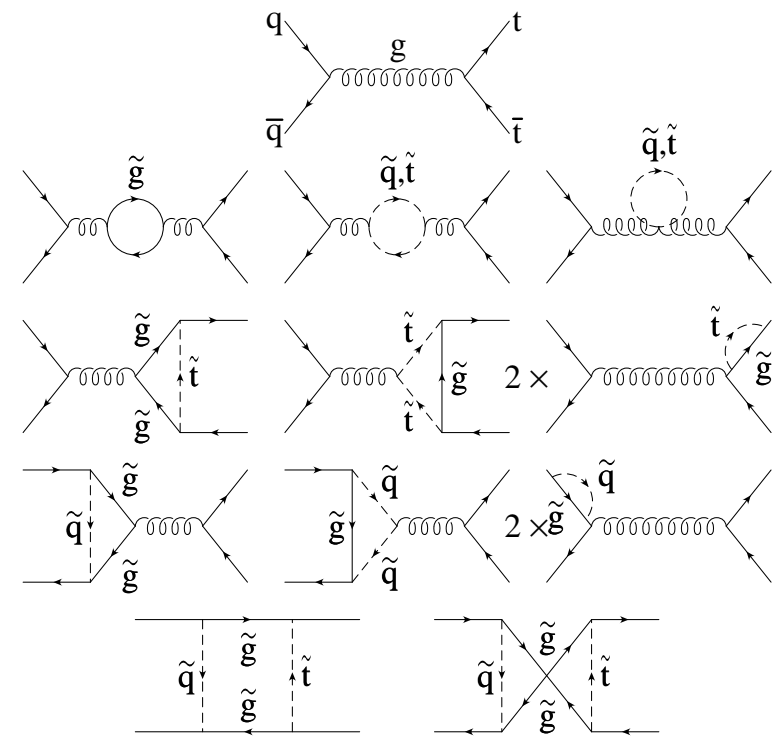

Figure 1:

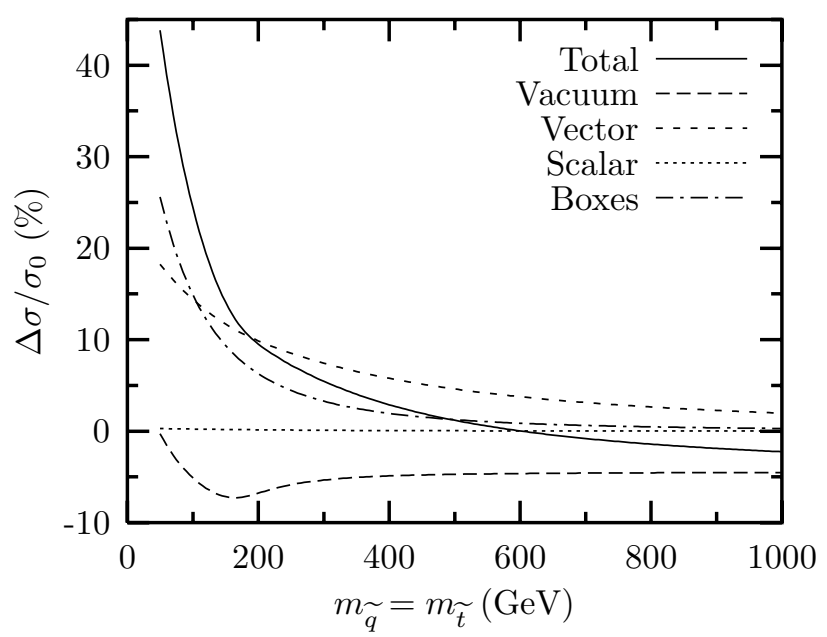

Figure 2: 


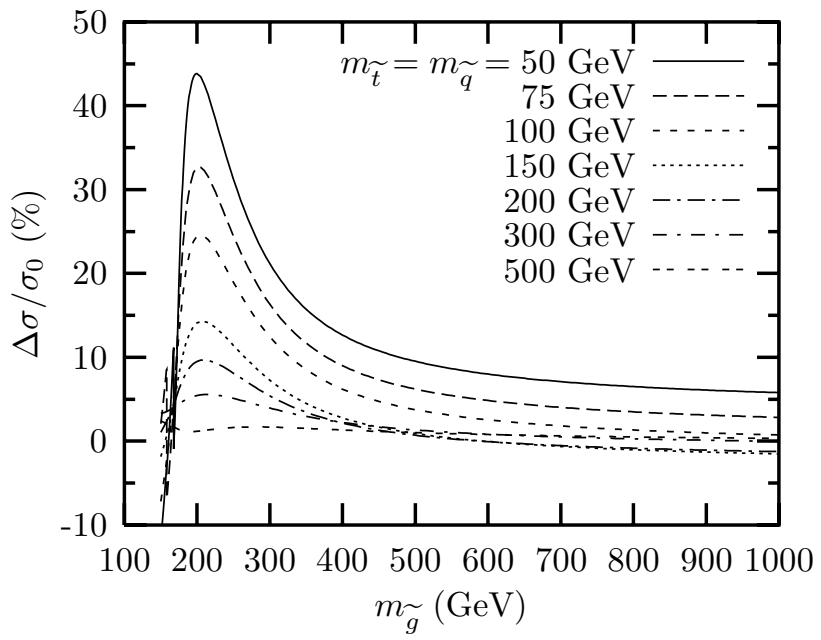

Figure 3:

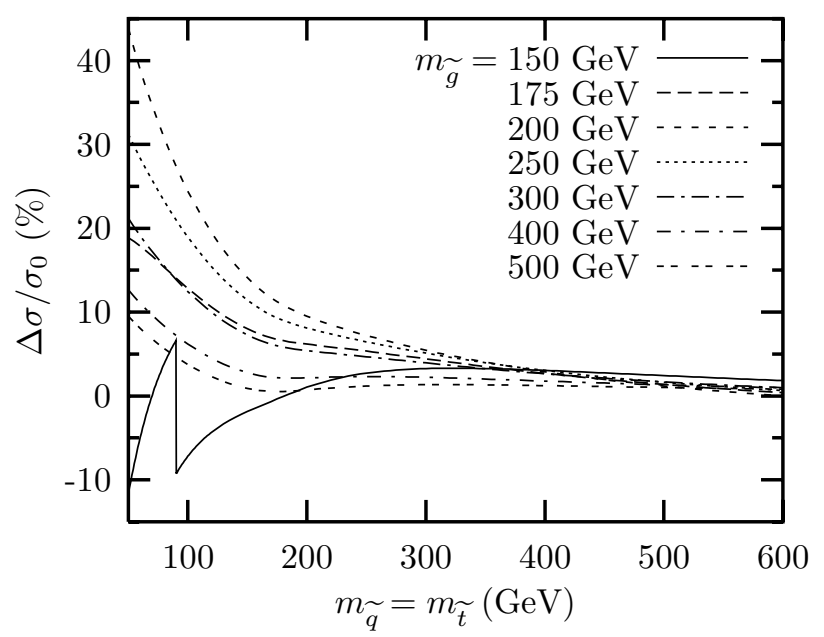

Figure 4: 


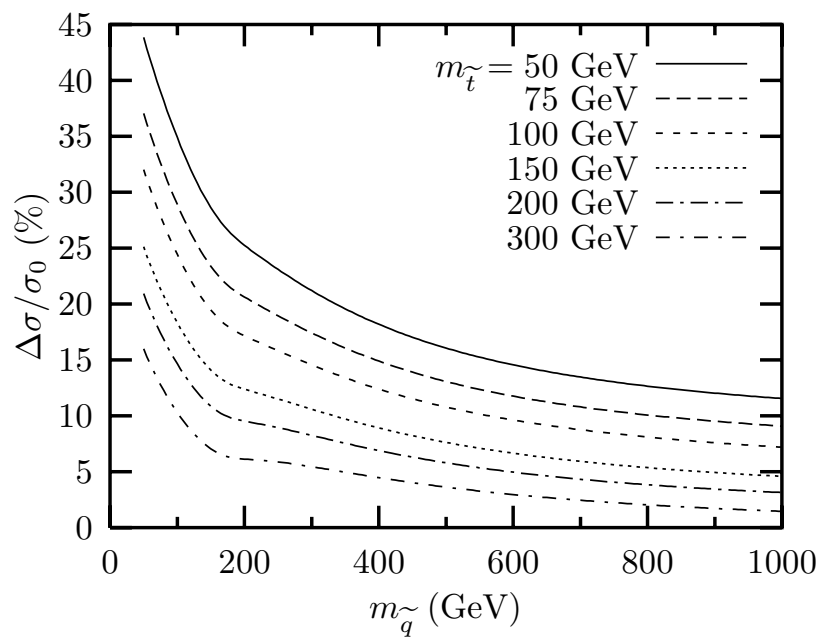

Figure 5:

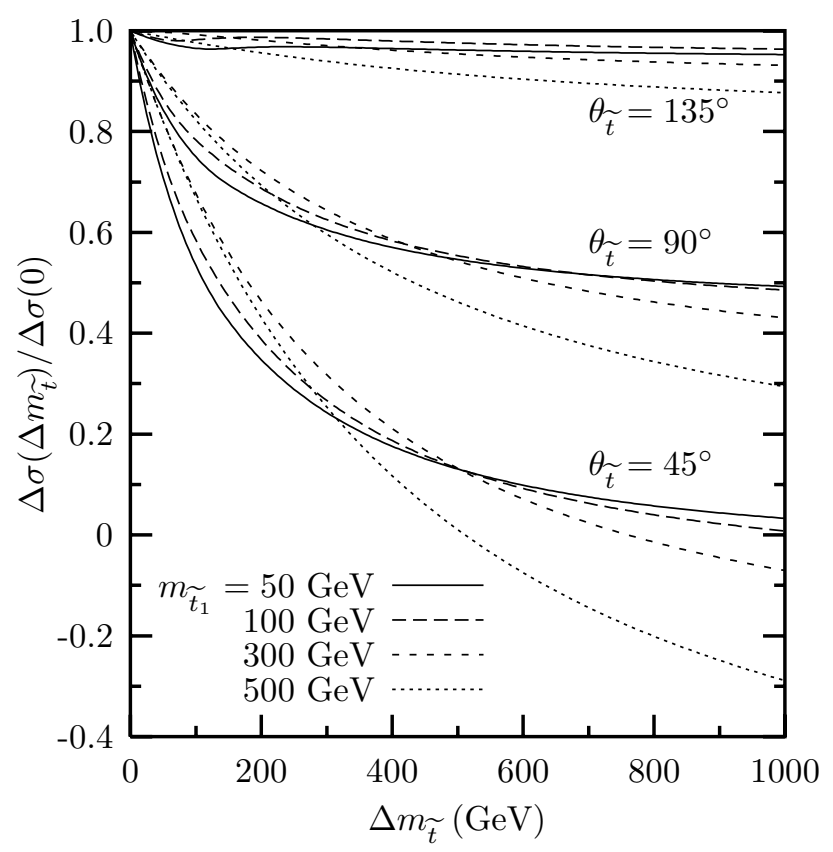

Figure 6: 


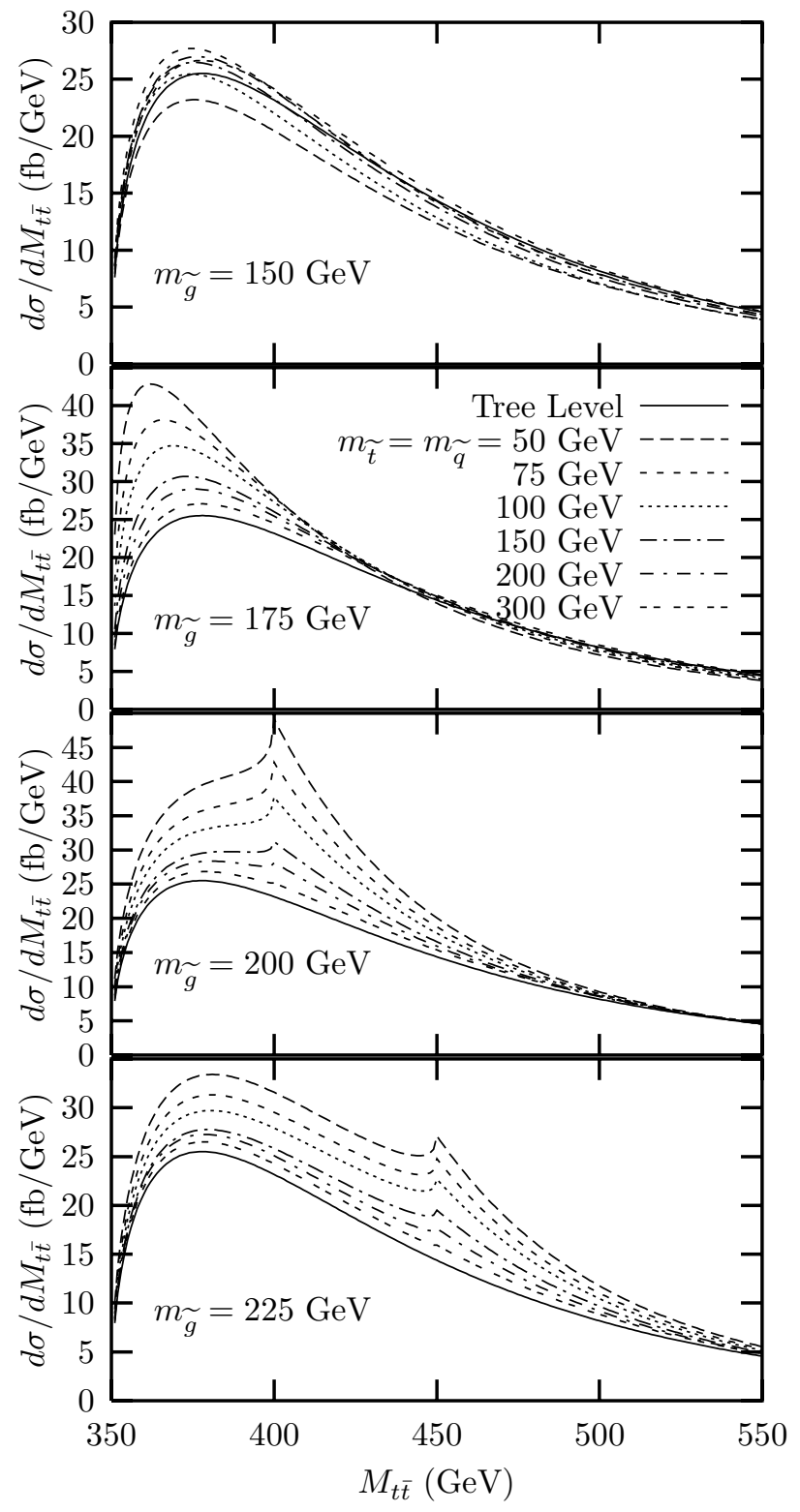

Figure 7: 